\title{
Manipulating Negative-Refractive Behavior with a Magnetic Field
}

\author{
Shiyang Liu, ${ }^{1,2}$ Weikang Chen, ${ }^{1}$ Junjie Du, ${ }^{1,2}$ Zhifang Lin, ${ }^{1,2}$ S. T. Chui, ${ }^{2}$ and C. T. Chan ${ }^{3}$ \\ ${ }^{1}$ Surface Physics Laboratory, Department of Physics, Fudan University, Shanghai 200433, China \\ ${ }^{2}$ Bartol Research Institute, University of Delaware, Newark, Delaware 19716, USA \\ ${ }^{3}$ Physics Department, Hong Kong University of Science and Technology, Clear Water Bay, Kowloon, Hong Kong \\ (Received 26 May 2008; revised manuscript received 8 September 2008; published 10 October 2008)
}

\begin{abstract}
We demonstrated a construction of negative-index material (NIM) with $\varepsilon_{\text {eff }}=\mu_{\text {eff }}=-1$ employing ferrites only, with no metallic components. Our design of the NIM is motivated by recent coherent potential approximation results and corroborated by exact numerical calculation demonstrating the negative refraction of an electromagnetic beam, with equal incident and refraction angles, as well as by the slab imaging phenomena, with the source-image separation twice as the slab thickness. The ferrite only based scheme furnishes the fabricated NIM with magnetically tunable working frequency, less loss and the air-matched wave impedance.
\end{abstract}

A material with simultaneously negative permittivity $\varepsilon$ and permeability $\mu$ exhibits many unique electromagnetic (EM) properties, including a negative-refractive index. The properties of the negative-index material (NIM) was theoretically predicted by Veselago [1] as early as in 1968. However, nature does not provide us with such materials. It is only fairly recently that NIMs are experimentally realized as man-made composites, and the interest in such materials surged immediately [2-6]. Intuitively, a negative response can be generated by the resonant excitation of individual scatterers, and thus negative effective permittivity and permeability can be obtained by mixing the negative electric and magnetic responses that come from different scatterers at a particular frequency. The relationship between the microscopic resonances and the macroscopic constitutive parameters $(\varepsilon$ and $\mu)$ can be obtained using effective medium theories [7]. Negative electric response can be typically obtained with metallic wires and strips [8] while a negative $\mu$ can be achieved through the low-frequency magnetic resonance of the split ring resonators [9]. Combining both class of resonators into a unit cell results in NIM, as was demonstrated experimentally [4-6]. Most of the NIMs that have been proposed and fabricated to date do rely on the idea of combining a component of negative $\varepsilon$ with another component of negative $\mu[2-6,10]$.

Quite recently, an alternative scheme was proposed based on the resonance in individual nonmagnetic dielectric scatterer with very high permittivity $[11,12]$. However, dielectrics with extremely high permittivity usually suffer from large damping. In addition, such resonant scatterers usually exhibit very different values of the permittivity and permeability near resonances; the NIMs so obtained typically show a high mismatch of the wave impedance with the air.

Most NIMs constructed usually display a narrow operational bandwidth, and the operational frequency is not tunable. To achieve external tunability of the negative- refractive index and a good impedance matching with air, we have proposed designs of the NIMs employing materials whose permittivity and/or permeability can be modulated by an external field. In this Letter, we report our designs of ferrite-based NIM, without the inclusion of any negative $\varepsilon$ component, nor negative $\mu$ constituent. Compared with conventional NIMs based on metallic resonant structures or nonmagnetic dielectric subwavelength resonators, our composite exhibits a magnetically tunable index (from positive to negative), air-matched wave impedance, as well as less absorption loss when appropriate ferrites are employed. In particular, a design of NIM with $\mu_{\text {eff }}=\varepsilon_{\text {eff }}=-1$ is demonstrated, with the verification by exact numerical simulation showing the negative refraction and the slab imaging effect.

We exemplify the ferrite only based designs with the two-dimensional (2D) case, generalization to three dimensions is straightforward. Let us start with a simple question. Given a 2D system composed of parallel dielectric rods placed in the air, when the system is illuminated by the transverse magnetic (TM) wave with the electric field polarized along the rod axis, under what condition can the system exhibit negative-index behavior? In Fig. 1, we show, at typical filling fraction $f=0.385$, the phase diagram of the system as a function of the permittivity $\varepsilon_{s}$ and the permeability $\mu_{s}$ for the rods based on the coherentpotential approximation (CPA) [7,13,14]. Above each curve is the region for $\varepsilon_{s}$ and $\mu_{s}$ that the system may simultaneously display negative electric and magnetic responses within a certain frequency range that satisfies $x=$ $(\omega / c) r_{s} \leq x_{s}$. Here, $\omega$ is the angular frequency, $r_{s}$ the rod radius, and $c$ the speed of light in vacuum. Three different curves are for three typical values of $x_{s}$. A further increase of permittivity above the curve usually produces a wider operational frequency bandwidth for the NIM, while a smaller $f$ demands a larger $\varepsilon_{s}$ to accomplish the negative index. The designs proposed in $[11,12]$ correspond to the special nonmagnetic case with $\mu=1$ in Fig. 1, where the 


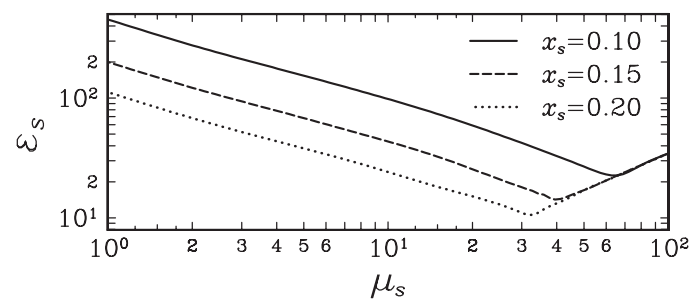

FIG. 1. Phase diagram for the permittivity $\varepsilon_{s}$ and permeability $\mu_{s}$ of dielectric rod of radius $r_{s}$. Above each curve is a region where the system may simultaneously display negative magnetic and electric responses at certain frequency regime satisfying $(\omega / c) r_{s} \leq x_{s}$.

requisite high permittivity of the building block to realize NIM is provided by ferroelectric ceramics and SiC, respectively [15], and the negative index is obtained within a fixed narrow operational frequency range. To relax the requirement for the extremely high permittivity (over 100 to generate NIM, as shown in Fig. 1) that is usually associated with large damping, and to overcome tunability constraints on the working frequency, we choose the ferrite as the building block, whose magnetically tunable permeability serves to achieve manipulability of the operational frequency range and the wave impedance. Furthermore, appropriate ferritelike single-crystal yttrium-iron garnet (YIG) displays a negligible low damping [16-18], so a NIM with low loss can be expected.

In our design, the ferrite rods are arranged in air as a square lattice with lattice constant $a$ and the rod axes along the $z$ direction. When fully magnetized, the ferrite has the magnetic permeability tensor [17]

$$
\begin{aligned}
\hat{\mu} & =\left(\begin{array}{ccc}
\mu_{r} & -i \mu_{\kappa} & 0 \\
i \mu_{\kappa} & \mu_{r} & 0 \\
0 & 0 & 1
\end{array}\right), \\
\mu_{r} & =1+\frac{\omega_{m}\left(\omega_{0}-i \alpha \omega\right)}{\left(\omega_{0}-i \alpha \omega\right)^{2}-\omega^{2}}, \\
\mu_{\kappa} & =\frac{\omega_{m} \omega}{\left(\omega_{0}-i \alpha \omega\right)^{2}-\omega^{2}},
\end{aligned}
$$

where $\alpha$ is the damping coefficient, $\omega_{0}=2 \pi \gamma H_{0}$ is the resonance circular frequency with $\gamma=2.8 \mathrm{MHz} / \mathrm{Oe}$ the gyromagnetic ratio, $H_{0}$ the sum of the external static magnetic field (ESMF) applied in $z$ direction and the shape anisotropy field [17], and $\omega_{m}=2 \pi \gamma M_{s}$ is the characteristic circular frequency with $M_{s}$ the saturation magnetization along $z$. In our in-principle demonstration, we set $\alpha=0$ [18] and $M_{s}=1750$ Oe, typical for YIG [17].

We have extended the CPA formulation $[13,14]$ for evaluating the effective permittivity $\varepsilon_{\text {eff }}$ and the effective permeability $\mu_{\text {eff }}$ to the case with gyrotropic scatterers, the details will be published elsewhere. In the limit $k_{e} r_{0} \ll 1$ and $k_{0} r_{0} \ll 1$, with $k_{e}=k_{0} \sqrt{\varepsilon_{\text {eff }}} \sqrt{\mu_{\text {eff }}}, k_{0}=\omega / c$, and $r_{0}=a / \sqrt{\pi}, \varepsilon_{\text {eff }}$ and $\mu_{\text {eff }}$ are governed by

$$
\varepsilon_{\mathrm{eff}}=(1-f) \varepsilon_{0}+f \tilde{\varepsilon}_{s}, \quad \frac{\mu_{\mathrm{eff}}-\mu_{0}}{\mu_{\mathrm{eff}}+\mu_{0}}=f \frac{\tilde{\mu}_{s}-\mu_{0}-\xi}{\tilde{\mu}_{s}+\mu_{0}+\xi}
$$

where the filling fraction $f=r_{s}^{2} / r_{0}^{2}$,

$$
\begin{gathered}
\tilde{\varepsilon}_{s}=2 \varepsilon_{s} F_{2}\left(k_{s} r_{s}\right), \quad F_{2}(x)=J_{1}(x) /\left[x J_{0}(x)\right] \\
\tilde{\mu}_{s}=\mu_{s} G_{2}\left(k_{s} r_{s}\right), \quad G_{2}(x)=J_{1}(x) /\left[x J_{1}^{\prime}(x)\right] \\
\xi=-\frac{(1-f) \mu_{0}^{2}\left(\mu_{\kappa} / \mu_{r}\right)^{2}\left(\tilde{\mu}_{s} / \mu_{s}\right)^{2}}{(1-f) \mu_{0}+(1+f) \tilde{\mu}_{s}}
\end{gathered}
$$

with $k_{s}=k_{0} \sqrt{\mu_{s}} \sqrt{\epsilon_{s}}, \mu_{s}=\left(\mu_{r}^{2}-\mu_{\kappa}^{2}\right) / \mu_{r}$ the effective permeability of the ferrite for the TM mode [17], $\varepsilon_{s}$ the ferrite permittivity, and $\varepsilon_{0}$ and $\mu_{0}$ the relative permittivity and permeability of the air background. When $\mu_{\kappa}=0$, $\xi=0$, Eq. (2) reduces to the isotropic case [13]. It is noted that for the gyromagnetic case, the phase boundary curves as shown in Fig. 1 are lower by a few percent for our designs with $\mu_{\kappa}^{\prime}=-\mu_{\kappa} /\left(\mu_{r}^{2}-\mu_{\kappa}^{2}\right)<0.1$, in favor of generating NIM with smaller requisite permittivity of the building block.

Figures 2(b) and 2(d) display $\varepsilon_{\text {eff }}$ and $\mu_{\text {eff }}$ as a function of the angular frequency $\omega$ for a square lattice of ferrite rods in the air with $a=10 \mathrm{~mm}$ and $r_{s}=3.5 \mathrm{~mm}$. The permeability of rod is given by Eq. (1) with the applied ESMF being such that $H_{0}=500 \mathrm{Oe}$ and $H_{0}=475 \mathrm{Oe}$, for Figs. 2(b) and 2(d), respectively. The permittivity of the ferrite rod is nondispersive $\varepsilon_{s}=25$ [18]. It is seen from Fig. 2(b) that within the frequency range from 15.5 to $16.2 \mathrm{GHz}$, the system displays simultaneously negative $\varepsilon_{\text {eff }}$ and $\mu_{\text {eff }}$ [19]. To confirm the result, photonic band structure is calculated using the rigorous frequencydomain multiple scattering theory [20]. The corresponding band diagrams are exhibited in Figs. 2(a) and 2(c). Excellent correspondence is obtained. In the frequency regimes with $\varepsilon_{\text {eff }}>0$ and $\mu_{\text {eff }}>0$, there appear photonic
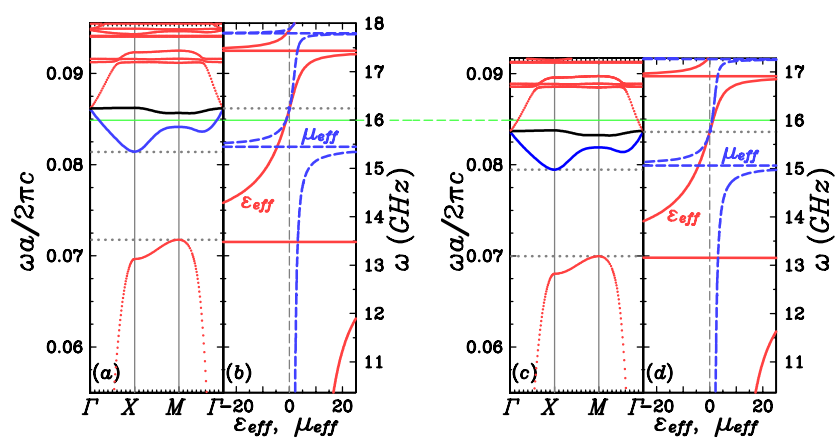

FIG. 2 (color online). (a) Photonic band structure of a square lattice of ferrite rods with lattice constant $a=10 \mathrm{~mm}$, rod radius $r_{s}=3.5 \mathrm{~mm}$, the saturation magnetization $M_{s}=1750 \mathrm{Oe}$, and $H_{0}=500$ Oe. (b) Effective permittivity $\varepsilon_{\text {eff }}$ (solid line) and effective permeability $\mu_{\text {eff }}$ (dashed line) versus circular frequency $\omega$, obtained from the CPA. (c) The same as (a) except $H_{0}=475$ Oe. (d) The same as (b) except $H_{0}=475$ Oe. 
bands in the band diagram, such as $\omega<13.5 \mathrm{GHz}$ in Figs. 2(a) and 2(b). While for $\varepsilon_{\text {eff }}<0$ and $\mu_{\text {eff }}>0$, a photonic band gap is opened up in the band diagram, as shown in Figs. 2(a) and 2(b) for $13.5 \mathrm{GHz}<\omega<$ $15.5 \mathrm{GHz}$ and $17.4 \mathrm{GHz}<\omega<17.7 \mathrm{GHz}$. Of particular interest is when $15.5 \mathrm{GHz}<\omega<16.2 \mathrm{GHz}$, where one has both $\varepsilon_{\text {eff }}<0$ and $\mu_{\text {eff }}<0$, implying an operational frequency range for the NIM. In the band diagrams, this corresponds to the second photonic band [as shown in Figs. 2(a) and 2(c)]. At about $16.2 \mathrm{GHz}$, one gets a socalled epsilon-near-zero (ENZ) medium [21], further investigation of which based on the same design will be published elsewhere. The comparison between the band diagram and $\varepsilon_{\text {eff }}$ and $\mu_{\text {eff }}$ demonstrates the validity of the CPA for gyromagnetic case $[13,14]$, based on which we found that at $\omega=16 \mathrm{GHz}$, denoted by the horizontal solid line in Figs. 2(a) and 2(b), $\varepsilon_{\text {eff }}=\mu_{\text {eff }}=-1$, resulting in an NIM with $n_{\text {eff }}=-1$.

To illustrate the tunability of the negative-refractive index by the ESMF, in Fig. 2(d), we show $\varepsilon_{\text {eff }}$ and $\mu_{\text {eff }}$ versus $\omega$ at a smaller ESMF such that $H_{0}=475$ Oe. The corresponding band diagram is given in Fig. 2(c). The photonic bands shift to lower frequency; so does the operational frequency range for the NIM. At $\omega=16 \mathrm{GHz}$, the refractive index is no longer negative. Instead, it is given by $n_{\text {eff }}=1.13$ with $\varepsilon_{\text {eff }}=0.92$ and $\mu_{\text {eff }}=1.4$. Therefore, by appropriately modulating the applied ESMF, the working wavelength for the NIM can be tuned, adding considerably to the flexibility in practical application in comparison with the conventional NIM constructs.

Figure 3 displays $\varepsilon_{\text {eff }}$ and $\mu_{\text {eff }}$ as a function of $H_{0}$ at the operating frequency $\omega=16 \mathrm{GHz}$. It can be seen that with the change of the ESMF by only a few percent, the system can be tuned from the NIM to conventional medium with simultaneously positive $\varepsilon_{\text {eff }}$ and $\mu_{\text {eff }}$.

To corroborate the theoretical design, we have simulated the propagation of a TM Gaussian beam through a wedge using the exact multiple scattering method at the working frequency $\omega=16 \mathrm{GHz}$. Typical results are shown in Figs. 4(a) and 4(b). The wedge is composed of 2788 ferrite rods arranged in the same structure and with the same rod parameters as in Fig. 2. The wedge angle is $\theta_{0}=30^{\circ}$. The beam shines on the interface normally from the left and enters the wedge, as shown in Fig. 4(a). The incident angle at the (right) wedge surface is $\theta_{\text {inc }}=\theta_{0}$. A negative re-

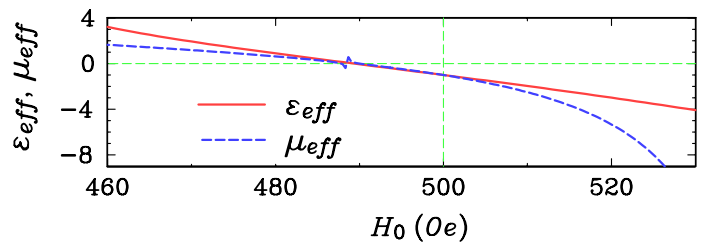

FIG. 3 (color online). Effective permittivity $\varepsilon_{\text {eff }}$ and permeability $\mu_{\text {eff }}$ versus $H_{0}$ at operating frequency $\omega=16 \mathrm{GHz}$, showing magnetically tunable negative-refractive index. fraction is clearly seen with the refraction angle $\theta_{\text {ref }} \approx$ $\theta_{\text {inc }}$, as shown in Fig. 4(a), corroborating the effective refractive index $n_{\text {eff }} \approx-1$. In addition, a very weak field appears at the bottom surface of the sample, suggesting that the reflection at the wedge surface is weak, offering persuasive evidence for the wave impedance match at both sides of the wedge interface. When $H_{0}$ is decreased to 475 Oe, however, the refractive index becomes positive from the CPA. The corresponding simulation results are shown in Fig. 4(b). The beam is seen to bend somewhat downwards, indicating a positive refraction, in agreement with the effective index $n_{\text {eff }}=1.13$ from the CPA.

The left-handed behavior of the system and its manipulability by the ESMF can also be visualized from the propagation of a Gaussian beam through a flat slab, which is typically presented in Figs. 4(c) and 4(d) for $H_{0}=500$ and $475 \mathrm{Oe}$, respectively. The system consists of 3634 ferrite rods with the same parameters as those in Fig. 4(a). A Gaussian beam, striking obliquely on the left surface of the slab with incident angle $\theta_{\text {inc }}=45^{\circ}$, is seen to refract negatively into the slab, with the refractive angle $\theta_{\text {ref }} \approx$ $\theta_{\text {inc }}$. Then it is subjected to another negative refraction at the right surface of the slab, as exhibited in Fig. 4(c), indicating once again the left-handed behavior with $n_{\mathrm{eff}} \approx$ -1 . The tunability of the left-handed behavior is shown in Fig. 4(d), with $H_{0}=475 \mathrm{Oe}$, where the beam goes through the slab without obvious change of its direction, consistent with positive effective index $n_{\text {eff }}=1.13$ from the CPA.

One of the most convincing characteristics for the NIM is the slab imaging effect [22]. Typical slab imaging phe-

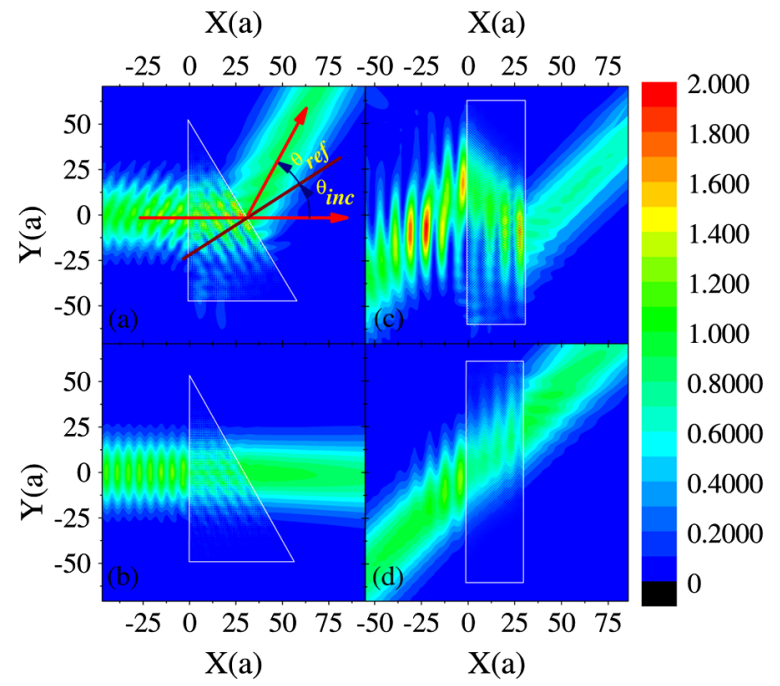

FIG. 4 (color online). A Gaussian beam shining on the wedge and slab samples is subjected to negative and positive refractions under different applied magnetic fields, with $H_{0}=500$ Oe (a) and (c), and $H_{0}=475 \mathrm{Oe}$ (b) and (d), showing magnetic tunability from negative to positive refraction. The sample boundaries are shown by the white lines and normal of the wedge surface by the black line. 


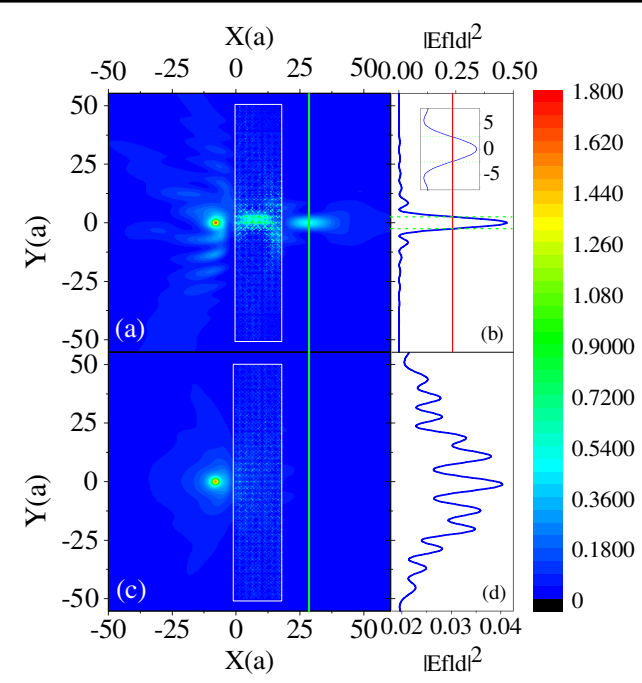

FIG. 5 (color online). Simulation on slab imaging phenomena for $H_{0}=500 \mathrm{Oe} \mathrm{(a)} \mathrm{and} H_{0}=475 \mathrm{Oe} \mathrm{(c).} \mathrm{(b)} \mathrm{and} \mathrm{(d)} \mathrm{give} \mathrm{the}$ intensity profile along the solid line in (a) and (c), respectively. The inset in (b) is an amplified part showing the image width at half maximum intensity. The slab boundaries are shown by the white lines.

nomena, together with its magnetically manipulability, based on our design of NIM is shown in Fig. 5, where the thickness of the slab is $t_{s}=18 a$. A monochromatic line source radiating at $\omega=16 \mathrm{GHz}$ is placed at a distance $d_{p}=8 a$ from the left surface of the slab. When $H_{0}=$ $500 \mathrm{Oe}$, an image is formed on the opposite side of the slab, centered at a distance $d_{i}=10.2 a$ from the right surface of the slab, as shown in Fig. 5(a). The profile of the field intensity along the solid line that goes through the image center is presented in Fig. 5(b), which reveals a transverse image size (width at half maximum intensity) $w \leqslant 5 a \approx 0.42 \lambda$, suggesting a possible subwavelength resolution. The separation $d$ between source and image is $d=d_{p}+d_{i}+t_{s}=36.2 a \approx 2 t_{s}$, consistent with negative-refractive index $n_{\text {eff }}=-1$. We have performed simulation with different values of $d_{p}$ ranging from $a$ to $12 a$, it is found that $d=2 t_{s}$ is satisfied to an accuracy of $1 \%$. This differs from the near-field imaging based on conventional photonic crystal, where $d_{i}$ has little dependence on $d_{p}$ [23]. It thus evidences a NIM with $n_{\text {eff }}=-1$. The magnetic tunability of negative-refractive index is exhibited in Figs. 5(c) and 5(d), where the only difference from Figs. 5(a) and 5(b) lies in that $H_{0}=475$ Oe instead of $500 \mathrm{Oe}$. The slab shows no left-handed behavior, and no image is formed on the opposite side of the slab.

In summary, based on the CPA, we have come up with the design of an NIM with $\varepsilon_{\text {eff }}=\mu_{\text {eff }}=-1$ by employing only ferrites, without the inclusion of any constituent of negative or extremely high permittivity. Our design has been corroborated by the exact numerical calculation demonstrating the negative refraction and the slab imaging phenomena. The ferrite only based NIM shows a magnetically tunable range of working frequency. In addition, the exclusion of extremely high and negative permittivity elements allows the construction of the NIM with the airmatched wave impedance and far less absorption loss, in favor of enhancing the transmitted signal.

This work was supported by CNKBRSF, NNSFC, PCSIRT, and Shanghai Science and Technology Commission. S. T. C. is partly supported by the US DOE. C. T. C. is supported by Hong Kong central allocation grant HKUST3/06C.

[1] V. G. Veselago, Sov. Phys. Usp. 10, 509 (1968).

[2] S. A. Ramakrishna, Rep. Prog. Phys. 68, 449 (2005).

[3] V. M. Shalaev, Nat. Photon. 1, 41 (2007).

[4] D. R. Smith et al., Phys. Rev. Lett. 84, 4184 (2000).

[5] R. A. Shelby et al., Appl. Phys. Lett. 78, 489 (2001).

[6] R. A. Shelby et al., Science 292, 77 (2001).

[7] P. Sheng, Introduction to Wave Scattering, Localization, and Mesoscopic Phenomena (Springer-Verlag, Berlin, 2006).

[8] J. B. Pendry et al., Phys. Rev. Lett. 76, 4773 (1996).

[9] J. B. Pendry et al., IEEE Trans. Microwave Theory Tech. 47, 2075 (1999); The split ring resonator was actually suggested decades earlier, see W. N. Hardy et al., Rev. Sci. Instrum. 52, 213 (1981); H. J. Schneider et al., ibid. 48, 68 (1977 ), but was made well known by Pendry et al. in generating negative magnetic response for NIM.

[10] F. J. Rachford et al., Phys. Rev. Lett. 99, 057202 (2007); Y.X. He et al., J. Magn. Magn. Mater. 313, 187 (2007).

[11] L. Peng et al., Phys. Rev. Lett. 98, 157403 (2007).

[12] J. A. Schuller et al., Phys. Rev. Lett. 99, 107401 (2007).

[13] Y. Wu et al., Phys. Rev. B 74, 085111 (2006).

[14] X. H. Hu et al., Phys. Rev. Lett. 96, 223901 (2006).

[15] For the system studied in [11], the phase diagram for $f=$ 0.16 (not shown here) suggests that the required value for $\varepsilon_{s}$ is over 520 , in agreement with [11] that employs the ferroelectric ceramic with permittivity $\varepsilon_{s} \approx 600$.

[16] Z. Wang et al., Phys. Rev. Lett. 100, 013905 (2008).

[17] D. M. Pozar, Microwave Engineering (Wiley, New York, 2004), 3rd ed.

[18] Single-crystal YIG can have a line width as low as 0.3 Oe, corresponding to $\alpha \sim 10^{-4}$. The loss tangent $\tan \delta$ is of order of $10^{-4}$, see [17], allowing neglect of damping.

[19] Note that in this frequency range, some terms in the permeability tensor are indeed negative, e.g., $\mu_{r}=$ -0.518 and $\mu_{\kappa}=2.76$ at $\omega=16 \mathrm{GHz}$ from Eq. (1). However, for the TM mode considered here, it is $\mu_{s}=$ $\left(\mu_{r}^{2}-\mu_{\kappa}^{2}\right) / \mu_{r}$ that plays the main role in describing electromagnetic wave propagation, see Ref. [17], and $\mu_{s}$ is positive in this frequency range. So our design is based on constituent with moderately large positive $\varepsilon$ and $\mu$.

[20] K. M. Leung and Y. Qiu, Phys. Rev. B 48, 7767 (1993).

[21] M. Silveirinha and N. Engheta, Phys. Rev. Lett. 97, 157403 (2006).

[22] J. B. Pendry, Phys. Rev. Lett. 85, 3966 (2000).

[23] Z. Y. Li and L. L. Lin, Phys. Rev. B 68, 245110 (2003). 